\title{
ESTADO DE HIDRATAÇÃO EM NADADORES APÓS TRÊS DIFERENTES FORMAS DE REPOSIÇÃO HÍDRICA NA CIDADE de Aracaju - SE
}

\author{
Eduardo Seixas Prado',2,3 espradoo@ig.com.br \\ Sheilla da Silva Barroso ${ }^{1,2}$ sheillassb@hotmail.com \\ Heline Oliveira Góis ${ }^{1,2}$ helinegois@hotmail.com \\ Thaise Reinert ${ }^{1,2}$ thaisereinert@hotmail.com
}

doi:10.3900/fpj.8.3.218.p

Prado ES, Barroso SS, Góis HO, Reinert T. Estado de hidratação em nadadores após três diferentes formas de reposição hídrica na cidade de Aracaju - SE. Fit Perf J. 2009 mai-jun;8(3):218-25.

\section{RESUMO}

Introdução: $O$ treinamento físico exige do atleta um bom estado de hidratação, pois a manutenção de níveis adequados de água pode favorecer o desempenho. O objetivo deste estudo foi avaliar o estado de hidratação de nadadores aracajuanos após sessões de treinamento com formas distintas de reposição hídrica. Materiais e Métodos: Oito nadadores $(24,25 \pm 7,30$ anos) realizaram três treinamentos controlados sob formas de reposição hídrica distintas: nenhum consumo de líquidos (GC), ingestão de água (GA), consumo de bebida esportiva (GS). O estado de hidratação foi determinado pela coloração urinária e percentual da perda da massa corporal. Resultados: Baseado na coloração da urina, GS demonstrou melhor classificação do estado de hidratação do que GC e GA. Diferença significativa foi encontrada em relação ao percentual da perda da massa corporal $(p<0,05)$ entre os grupos, com vantagem hídrica para GS. Discussão: A água pode promover um adequado estado de hidratação, mas bebida esportiva parece oferecer uma melhor reposição hídrica.

\section{PALAVRAS-CHAVE}

Natação, Ingestão de Líquidos, Desidratação.

\footnotetext{
1 Universidade Estadual de Londrina - UEL - Grupo de Estudo e Pesquisa em Atividade Física e Saúde - GEPAFIS - Londrina - Brasil

${ }^{2}$ Universidade Tiradentes - Unit - Departamento de Educação Física - Aracaju - Brasil

${ }^{3}$ Universidade Federal de Uberlândia - UFU - Instituto de Genética e Bioquímica - Uberlândia - Brasil
} 
Hydration status in swimmers after three different forms of fluid replacement from Aracaju city - SE BRAZIL

\section{ABSTRACT}

Introduction: The physical training of athletes requires a good state of hydration, because the maintenance of adequate levels of water can promote the performance. The objective of the study is to assess the hydration status of aracajuanos swimmers after training sessions with different forms of fluid replacement. Materials and Methods: Eight swimmers (age 24,25 7,30 years) completed three training controlled under different forms of fluid replacement: no fluid intake (GC), water intake (GA), consumption of sport beverage (GS). Hydration status was determined by urine color and percentage of body mass change. Results: Based on urine color, GS has better classification of hydration status than GC and GA. Significant difference was found in relation to percentage of body mass change $(p<0,05)$ between groups, with advantage fluid replacement for GS. Discussion: The water can promote an adequate hydration status, but sport beverage seems to offer a better fluid replacement.

\section{KEYWORDS}

Swimming, Drinking, Dehydration.

\section{ESTADO DE HIDRATACIÓN DE LOS NADADORES DESPUÉS DE TRES DIFERENTES FORMAS DE REPOSICIÓN HÍDRICA EM LA CIUdAd de Aracaju - SE - BrasiL}

\section{RESUMEN}

Introducción: El entrenamiento físico de los atletas requiere un buen estado de hidratación, porque el mantenimiento de niveles adecuados de agua puede favorecer el rendimiento físico. El objetivo és evaluar el estado de hidratación de los nadadores aracajuanos después de sesiones de entrenamiento con diferentes formas de reposición hídrica. Materiales y Métodos: Ocho nadadores $(24,25 \pm 7,30$ años) realizaron tres entrenamientos controlados sub diferentes formas de reposición hídrica: sin consumo de líquido (GC), ingestión de agua (GA), consumo de bebida deportiva (GS). El estado de hidratación se determinó mediante el color de la orina y el porcentaje de la pérdida de masa corporal. Resultados: Se basa en el color de la orina, GS tiene una mejor clasificación del estado de hidratación que en GC y GA. Se encontraron diferencias significativas en el porcentaje de la pérdida de masa corporal $(P<0,05)$ entre los grupos con ventaja hídrica para GS. Discusión: El agua puede promover un adecuado estado de hidratación, pero bebida deportiva parece ofrecer una mejor reposición hídrica.

\section{PALABRAS CLAVE}

Natación, Ingestión de Líquidos, Deshidratación.

\section{INTRODUÇÃO}

Manter-se hidratado antes, durante e após o exercício físico é essencial para atletas, que podem ter seu desempenho prejudicado caso não haja ingestão suficiente de algum líquido $\mathbf{1 , 2 , 3 , 4}$.

Sabe-se que a reposição hídrica adequada regula a circulação sanguínea, o volume plasmático e o controle da temperatura corporal. Não compensar a perda de líquidos, principalmente no exercício físico em ambientes quentes e úmidos, pode trazer sérias consequências ${ }^{5,6}$. Um estado de hidratação inadequado limita efetivamente a manutenção da temperatura a tal ponto que a termorregulação é afetada, determinando respostas fisiológicas, como decréscimo no desempenho, danos termais e, em casos severos, até mesmo a morte ${ }^{\mathbf{3 , 7 , 8 , 9}}$. Também pode afetar as respostas cardiovasculares no exercício físico, prejudicando o desempenho $7,10,11,12$. Durante um exercício físico aeróbico no calor a frequên- cia cardíaca aumenta e o volume de ejeção, o débito cardíaco e o fluxo sanguíneo para o músculo exercitado podem ser reduzidos, podendo levar a situação de "cardiovascular drift"13,14,15,16,17.

Na realização de exercícios físicos prolongados, associados a ambientes quentes e úmidos, a definição do tipo de líquido a ser ingerido é importante e contribuições importantes têm sido feitas ${ }^{18,19}$. A ingestão de água parece não ser a melhor alternativa de consumo líquido, mas sim bebidas esportivas ${ }^{20}$. Sugere-se a inclusão de eletrólitos, principalmente o sódio, aumentando a retenção de água no espaço intracelular ${ }^{21}$. Se o líquido ingerido incluir carboidrato, essa melhora é mais significativa, especialmente em exercícios físicos prolongados com sudorese intensa $\mathbf{2 2}^{\mathbf{2 2}}$. Porém, críticas a tais recomendações foram publicadas recentemente ${ }^{\mathbf{2 3}}$.

Além do tipo de bebida, a caracterização das práticas e a avaliação do estado de hidratação constituem 
elementos importantes para garantir uma hidratação

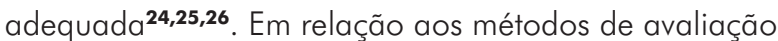
do estado de hidratação, várias formas são utilizadas atualmente ${ }^{\mathbf{2 7}, 28}$. A massa corporal e a coloração urinária constituem técnicas simples de avaliação da hidratação que podem ser utilizadas com segurança para tal finalidade $27,29,30,31,32$

A cidade de Aracaju é caracterizada por um clima quente e úmido, que pode proporcionar prejuízos no desempenho físico dos atletas locais durante o período de treinamento, independente da modalidade esportiva. A natação, apesar de praticada em ambiente aquático, também pode provocar tais danos. No entanto, ainda são desconhecidos os níveis de hidratação dos nadadores aracajuanos. Portanto, o objetivo deste estudo foi verificar o estado de hidratação em atletas de natação da cidade de Aracaju, submetidos a diferentes formas de reposição hídrica durante sessões de treinamento.

\section{MATERIAIS E MÉTODOS}

A amostra foi composta por oito atletas $(24,25 \pm 7,30$ anos) do sexo masculino, participantes de competições nacionais há pelo menos cinco anos, voluntários e residentes no município de Aracaju - SE. Nenhum participante tinha histórico médico com problemas de saúde e não usavam substâncias ergogênicas ou qualquer outro tipo de droga que pudesse alterar o resultado do estudo. Todos foram informados e orientados com antecedência sobre a realização do estudo e assinaram um termo de consentimento livre e esclarecido, o qual garantiu a privacidade de informações pessoais. $\bigcirc$ presente trabalho também atendeu às normas para a realização de pesquisa em seres humanos, resolução 196 do Conselho Nacional de Saúde de 10/10/96, e foi aprovado pelo Comitê de Ética em Pesquisa da Universidade Tiradentes (UNIT/SE), sob número $160907 R$.

Durante todo o estudo os atletas realizaram, no período de 12 dias, três treinamentos com ingestão de líquidos controlada (TC), em dias diferentes, com intervalos de $72 \mathrm{~h}$
Três dias antes do primeiro TC $\left(D_{1-3}\right)$, o estudo foi iniciado. Nesse período, os atletas foram instruídos a manter sua alimentação rotineira e treinamento normal (TN), mas que fosse ingerido somente água ou bebidas descafeinadas, ad libitum, como dieta líquida (DL). $\bigcirc$ mesmo foi repetido entre o $5^{\circ}$ e $7^{\circ}$ dias $\left(D_{5-7}\right)$ e o $9^{\circ}$ e $11^{\circ}$ dias $\left(D_{9-11}\right)$.

No primeiro dia de TC ou $4^{\circ}$ dia de experimento $\left(D_{4}\right)$, ninguém consumiu água e/ou outro tipo de líquido durante o treinamento, sendo esse grupo denominado de controle (GC). No segundo TC ou $8^{\circ}$ dia $\left(D_{8}\right)$, os atletas consumiram apenas água mineral da marca Minalba ${ }^{\circledR}$ durante o treino, que foi denominado de grupo água (GA). No terceiro TC ou $12^{\circ}$ dia $\left(D_{12}\right)$, os atletas ingeriram uma bebida esportiva (solução de carboidrato e eletrólitos) da marca Gatorade ${ }^{\circledR}$ durante o treino, denominado de grupo suplemento (GS). Vale ressaltar que no $D_{4}, D_{8}$ e $D_{12}$ os atletas mantiveram as recomendações da DL nos momentos anteriores e posteriores ao TC, e que o controle acima mencionado ocorreu somente durante o TC, com os atletas ingerindo suas respectivas bebidas ad libitum. Além disso, os treinos foram iguais, em duração e intensidade, para todos os atletas nos dias de TC.

Em cada TC, tanto antes como depois, os seguintes dados foram coletados: temperatura ambiente (em graus centígrados, ${ }^{\circ} \mathrm{C}$ ), umidade relativa do ar (em percentual, \%), duração do treino (em minutos, min), distância percorrida (em metros, m), consumo médio de líquidos (em mililitros, $\mathrm{mL}$ ), massa corporal (em quilograma,kg) e coloração urinária. Os dois últimos foram utilizados como marcadores simples para avaliação do estado de hidratação.

A massa corporal foi registrada antes (pré) e após (pós) cada TC, utilizando-se para tal uma balança antropométrica da marca Filizola, com precisão de $100 \mathrm{~g}$. No momento da medida os indivíduos estavam em pé, de frente para o avaliador, na posição ereta, pés afastados à largura do quadril, descalços e usando roupas leves.

As amostras urinárias foram coletadas pelos próprios atletas, inicialmente ao acordar pela manhã (pré), e imediatamente após (pós) todos os TC (Figura 1). Logo depois, a urina coletada foi entregue para análise e de-

Figura 1 - Desenho experimental. A escala de tempo está em dias (D) e os detalhes do protocolo são mostrados. Antes de cada dia de treinamento controlado (TC), os atletas foram submetidos a uma dieta líquida (DL) e treinamento normal (TN) como descrito nos métodos $\left(D_{1-3} ; D_{5-7} ; D_{9-11}\right)$. Urina (U) e massa corporal (MC) foram coletadas antes (pré) e depois (pós) do TC sob diferentes formas de reposição hídrica (nenhum consumo de líquidos, ingestão de água, consumo de bebida esportiva) nos dias $D_{4}, D_{8}$ e $D_{12}$.

\begin{tabular}{lccccc}
\hline $\mathrm{DL}$ e TN & $\begin{array}{c}\mathrm{TC} \text { sem líquidos; } \mathrm{MC} \text { e U } \\
\text { (pré e pós) }\end{array}$ & $\mathrm{DL}$ e TN & $\begin{array}{c}\mathrm{TC} \text { com água; } \mathrm{MC} \text { e } \mathrm{U} \\
\text { (pré e pós) }\end{array}$ & DL e TN & $\begin{array}{c}\mathrm{TC} \text { com bebida esportiva; } M C \text { e } \mathrm{U} \\
\text { (pré e pós) }\end{array}$ \\
\hline $\mathrm{D}_{1-3}$ & $\mathrm{D}_{4}$ & $\mathrm{D}_{5-7}$ & $\mathrm{D}_{8}$ & $\mathrm{D}_{9-11}$ & $\mathrm{D}_{12}$ \\
\hline
\end{tabular}


terminação de sua coloração, tendo como referência a escala de Armstrong et al. ${ }^{29}$. As amostras mantiveram-se em temperatura ambiente até serem analisadas, imediatamente após as coletas. Os resultados obtidos da massa corporal e coloração urinária foram classificados perante a tabela proposta por Casa et al. ${ }^{33}$.

Inicialmente, os dados foram expressos como média e desvio padrão. Em seguida, foi realizado o teste para determinação do grau de homogeneidade da amostra. Quando a amostra apresentou uma distribuição normal, foi aplicada a análise de variância (ANOVA) One Way para verificação das diferenças entre as médias dos índices da coloração urinária, massa corporal e percentual da diferença da massa corporal dos grupos. Quando a amostra não demonstrou normalidade, foi utilizado o teste de Kruskal-Wallis One Way. Todas as formas de verificação das diferenças entre as médias foram combinadas ao teste post-hoc de Tukey, adotando-se um nível de significância de 5\%. Os resultados da classificação do estado de hidratação obtidos do índice da coloração urinária também foram expressos como percentual (\%).

\section{RESULTADOS}

As condições ambientais, a duração, a distância percorrida nos treinos, bem como o consumo de água e bebida esportiva, estão descritos na Tabela 1. Durante os TC a temperatura situou-se entre $28^{\circ} \mathrm{C}$ e $29^{\circ} \mathrm{C}$, com uma umidade relativa de $66 \%, 62 \%$ e $65 \%$, respectivamente no $D_{4}, D_{8}$ e $D_{12}$. A distância percorrida foi de $3.100 \mathrm{~m}$, com uma duração de 80 min em todos os TC, com um consumo médio de água de $575,00 \pm 345,38 \mathrm{~mL}$ e de bebida esportiva de $400,00 \pm 297,61 \mathrm{~mL}$.

Não foram encontradas diferenças significativas entre os grupos para os índices de coloração urinária $(p>0,05)$ (Tabela 2). No entanto, todos os grupos apresentaram índices elevados, tanto antes $\left(\mathrm{GC}_{\text {pré }}=4,50 \pm 1,92 ; \mathrm{GA}_{\text {pré }}=4,87 \pm 1,45\right.$ e $\left.G S_{\text {pré }}=5,00 \pm 1,19\right)$ quanto depois dos $\mathrm{TC}\left(\mathrm{GC}_{\text {pós }}=5,62 \pm 1,59 ; \mathrm{GA}_{\text {pós }}=5,62 \pm 1,76 \mathrm{e}\right.$ $\left.\mathrm{GS}_{\text {pós }}=5,00 \pm 2,26\right)$. Tal resultado demonstra que os grupos já apresentavam algum estado de desidratação nos momentos que antecederam os TC. Além disso, os valores elevados dos índices apresentados após os TC caracterizam um estado de hidratação precário, independentemente do recurso líquido utilizado.

Por outro lado, embora não sejam demonstradas diferenças significativas em relação aos valores absolutos dos índices de coloração urinária, mas sim a verificação de um estado de desidratação entre os três grupos, especialmente no momento pós TC, independentemente do recurso líquido ingerido, o GS promoveu uma melhor hidratação em comparação com GC e GA, quando uma análise percentual da classificação do estado de hidratação dos atletas foi realizada em relação à coloração urinária.

No GC ${ }_{\text {pré' }} 62 \%$ dos atletas estavam eu-hidratados ou com desidratação mínima, enquanto $38 \%$ encontravamse entre uma desidratação significativa ou grave. No momento em que o $\mathrm{GC}_{\text {pós }}$ foi analisado, este apresentou um aumento no percentual de atletas com desidratação significativa ou grave de $62 \%$ para $75 \%$, e consequentemente, o percentual de eu-hidratação diminuiu de 38\% para $25 \%$, ou seja, houve uma piora na classificação do estado de hidratação (Figura 2A).

Tabela 1 - Caracterização do controle amostral

\begin{tabular}{lcccccc}
\hline dias TC & $\begin{array}{c}\text { temperatura } \\
\left({ }^{\circ} \mathrm{C}\right)\end{array}$ & $\begin{array}{c}\text { umidade } \\
(\%)\end{array}$ & $\begin{array}{c}\text { duração treino } \\
(\mathrm{min})\end{array}$ & $\begin{array}{c}\text { distância } \\
\text { percorrida } \\
(\mathrm{m})\end{array}$ & $\begin{array}{c}\text { consumo médio de água } \\
\pm \text { desvio padrão } \\
(\mathrm{mL})\end{array}$ & $\begin{array}{c}\text { consumo médio da bebida } \\
\text { esportiva } \pm \text { desvio padrão } \\
(\mathrm{mL})\end{array}$ \\
\hline $\mathrm{D}_{4}$ & 28 & 66 & 80 & 3100 & - & - \\
$\mathrm{D}_{8}$ & 28 & 62 & 80 & 3100 & $575 \pm 345,38$ & - \\
$\mathrm{D}_{12}$ & 29 & 65 & 80 & 3100 & - & $400 \pm 297,61$ \\
\hline
\end{tabular}

Tabela 2 - Valores absolutos da massa corporal e índice de coloração urinária antes (pré) e depois (pós) do TC, expressos como média \pm desvio padrão.

\begin{tabular}{llcc}
\hline grupos & & massa corporal $(\mathrm{kg})$ & índice de coloração urinária \\
\hline \multirow{2}{*}{ GC } & pré & $84,84 \pm 8,36$ & $4,50 \pm 1,92$ \\
& pós & $83,41 \pm 8,03$ & $5,62 \pm 1,59$ \\
GA & pré & $84,36 \pm 8,38$ & $4,87 \pm 1,45$ \\
& pós & $83,78 \pm 8,29$ & $5,62 \pm 1,76$ \\
GS & pré & $84,35 \pm 8,05$ & $5,00 \pm 1,19$ \\
& pós & $83,80 \pm 7,80$ & $5,00 \pm 2,26$ \\
\hline
\end{tabular}

Nenhuma diferença entre os grupos para todas as variáveis $(p>0,05)$ 
Alterações semelhantes ao GC foram verificadas

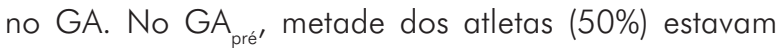
eu-hidratados ou com desidratação mínima, e a outra metade (50\%) demonstrava desidratação significativa ou grave. No momento em que o $\mathrm{GA}_{\text {pós }}$ foi analisado, o percentual de atletas eu-hidratados ou com desidratação

Figura 2 - Percentual da classificação do estado de hidratação pela coloração urinária dos atletas nos momentos pré e pós treinamento nos grupos GC (A), GA (B) e GS (C) (A)

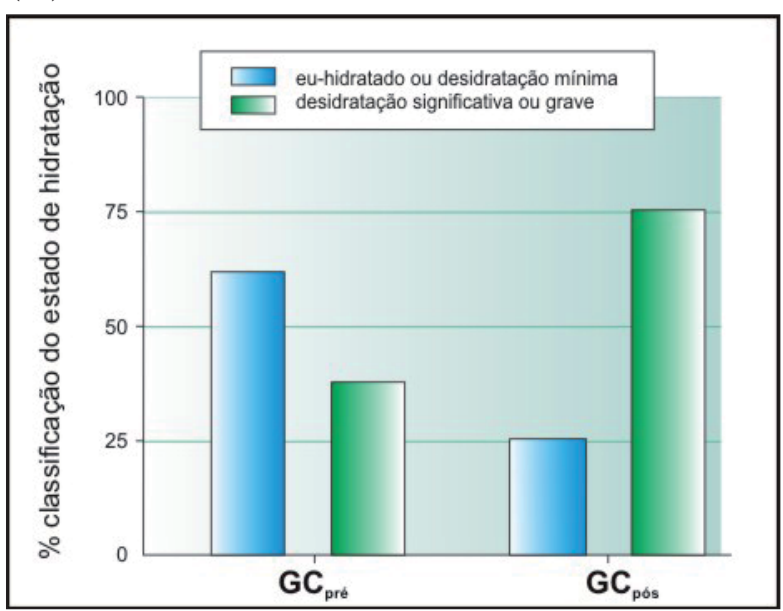

(B)

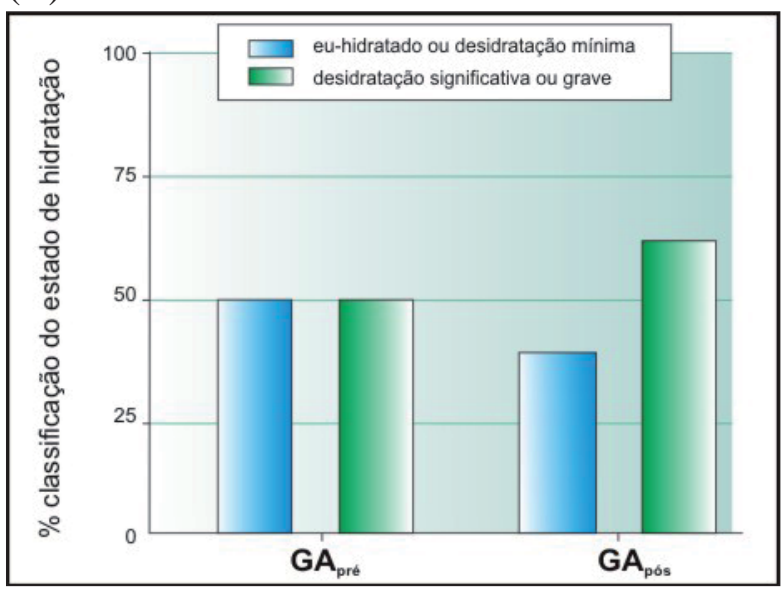

(C)

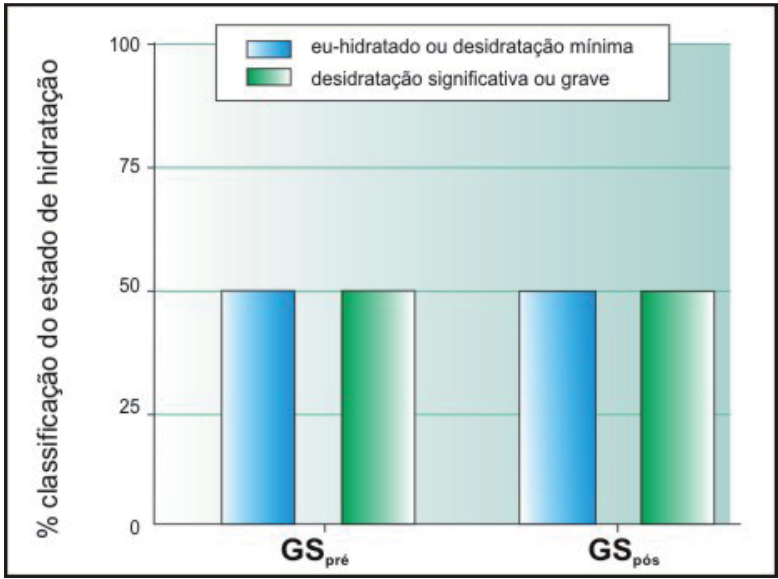

mínima diminuiu para 38\%, assim como o percentual de desidratação significativa ou grave se elevou para $62 \%$, ou seja, também houve um prejuízo na classificação do estado de hidratação (Figura 2B).

Diferentemente dos outros grupos, o GS não alterou seu percentual de classificação do estado de hidratação. Tanto no GS pré quanto no $\mathrm{GS}_{\text {pós' }}, 50 \%$ dos atletas estavam eu-hidratados ou com desidratação mínima, e a outra metade (50\%) com desidratação significativa ou grave (Figura 2C). A manutenção do percentual de classificação evidencia um melhor estado de hidratação neste grupo.

Quanto aos valores absolutos da massa corporal, nenhuma diferença significativa foi observada entre os grupos $(p>0,05)$ (Tabela 2$)$. Porém, analisando a variação do percentual da diferença da massa corporal, tanto GC como GA, demonstraram perdas percentuais de massa corporal (-1,66\% e -0,69\%, respectivamente), enquanto que em GS foi verificado um ganho ( $+0,72 \%)$. No entanto, isso não representou uma diferença significativa entre GA e GS. Houve diferença quanto a esse percentual entre os grupos GA e GC, e entre GA e GC $(p<0,05)$ (Figura 3).

\section{DISCUSSÃO}

Sabe-se que a temperatura, umidade, intensidade, duração do exercício, adaptação do atleta ao meio, perda de fluidos, além de outros fatores, devem ser levados em consideração para adoção adequada de um regime de hidratação antes, durante e após exercícios físicos ${ }^{34}$. Em temperaturas e umidades elevadas, o desempenho em exercícios prolongados é reduzido, podendo levar à fadiga $^{35}$

No estudo de Binkley et al. ${ }^{36}$ consta-se que altas temperaturas durante um exercício físico levam a um risco alto de exaustão, de estresse e de possíveis danos à vida do atleta. Murray ${ }^{37}$ também relata que os prejuízos desencadeados por uma hidratação inadequada, em temperaturas elevadas, prejudicam o desempenho na competição, provocando riscos à saúde. Para pessoas que vivem ou competem nesses ambientes, recomendase uma boa dieta líquida, consumindo quantidades adequadas de fluidos durante as $24 \mathrm{~h}$ que precedem o evento, especialmente durante o período entre a última alimentação e o exercício, para que seu rendimento não seja prejudicado ${ }^{38}$.

Recomenda-se beber aproximadamente $500 \mathrm{~mL}$ de líquido, cerca de $2 \mathrm{~h}$ antes do exercício, para promover uma hidratação adequada e permitir que haja tempo para excretar o excesso de água ingerido. Além disso, aconselha-se um aumento da hidratação nas pausas do treino, como também a ingestão de $1 \mathrm{~L}$ a 1,25L de líquido 
para cada quilograma de água corporal perdido durante o exercício ${ }^{38,34}$. Dessa maneira, é possível prevenir uma desidratação ou provável hipertermia, bem como decréscimos no desempenho durante o exercício ${ }^{8,3}$. Geralmente, a cidade de Aracaju apresenta a umidade relativa do ar e temperatura ambiental elevada $(\sim 80 \%$ e $\sim 35^{\circ} \mathrm{C}$, respectivamente). No entanto, nesse estudo, tais variáveis mantiveram-se relativamente baixas e, mesmo assim, foi possível observar danos ao estado de hidratação.

Baseado nos índices de coloração urinária, antes dos TC todos os grupos já estavam desidratados. Esta ocorrência deve-se ao fato dos atletas não se hidratarem adequadamente, levando a um estado de desidratação cumulativa e progressiva. A desidratação pode ser decorrente tanto da sudorese intensa durante a prática do exercício quanto de uma ingestão insuficiente e/ou uma deficiência na absorção de líquidos. Por isso, é importante reconhecer os elementos que influem na qualidade da hidratação ${ }^{6}$. Ao atingir um nível de desidratação grave, o atleta já está desidratado antes mesmo de iniciar o treinamento ou está se hidratando inadequadamente durante e após os treinos, de modo que ele também inicia o treino subsequente com um estado de hidratação baixa ${ }^{39}$.

Quando as recomendações de hidratação no exercício não são seguidas, os níveis de desidratação parecem ocorrer antes do treino ou competição. Kutlu \& Guler ${ }^{40}$ observaram um estado de desidratação em atletas de tae-kwon-do através da coloração urinária pela manhã. Outro estudo, investigando o estado de hidratação pré-jogo em ambiente seco e quente, de atletas de futebol, voleibol e basquetebol da Austrália, através da gravidade específica da urina e coloração urinária, observou que $6 \%$ dos atletas estavam com desidratação grave, $50 \%$ com desidratação significativa, $31 \%$ com desidratação mínima e 14\% eu-hidratados ${ }^{41}$.

Os índices de coloração urinária observados após os TC, também demonstraram um estado de desidratação dos atletas, independentemente da solução ingerida. Parece que a deficiente reposição de líquidos não é fato isolado apenas entre os atletas de natação da cidade de Aracaju. Segundo Armstrong et al. ${ }^{38}$, o volume de fluidos que a maioria dos atletas ingere voluntariamente durante o treinamento não repõe $100 \%$ do fluido perdido no suor, principalmente em exercícios de alta intensidade num ambiente de temperatura elevada, já que nestas condições a taxa de suor e o volume de fluidos perdidos aumentam. Yeargin et al. ${ }^{\mathbf{2}}$ também detectaram um estado de desidratação em jogadores de futebol americano avaliados pela coloração urinária, durante oito dias de treinamento.
Do mesmo modo, Tan \& Sunaria ${ }^{43}$, verificaram um estado de desidratação em atletas velejadoras durante uma competição.

Uma observação importante nesse estudo foi em relação ao percentual da classificação do estado de hidratação, baseada na coloração urinária, em euhidratação, desidratação mínima, desidratação significativa e desidratação grave, proposto por Casa et al. ${ }^{33}$. Nadadores no GS $_{\text {pós }}$ sofreram menor prejuízo hídrico em relação aos outros grupos quando uma análise do percentual dessa classificação foi realizada, ou seja, um número menor de atletas se encontrava no estado de desidratação significativa ou grave após o TC. Porém, em relação ao exposto acima, vale ressaltar alguns pontos interessantes.

É importante salientar que ainda não há um consenso na comunidade científica quanto aos métodos ideais de avaliação do estado de hidratação, inclusive existem dúvidas quanto ao uso dos índices de coloração urinária para tal. Armstrong et al. ${ }^{30}$, investigando a validade e a sensibilidade da coloração urinária como índice do estado de hidratação, observaram em ciclistas treinados, do sexo masculino, que a coloração urinária é um índice válido para avaliar o estado de hidratação, podendo ser utilizada tão bem, ou melhor, que outros marcadores. Kavouras ${ }^{44}$ também reconhece a coloração urinária como um marcador efetivo do estado de hidratação. Parece que a soma de mais de um marcador simples do estado de hidratação é o suficiente para uma avaliação precisa. $\bigcirc$ uso da coloração urinária, unicamente, pode não ser um marcador de estado de hidratação após 6 h do término do exercício ${ }^{45}$

Outro ponto é que existem controvérsias acerca da eficácia do consumo de bebidas esportivas, bem

\section{Figura 3 - Percentual da diferença de massa corporal entre os grupos. Os valores são expressos como média \pm desvio padrão}

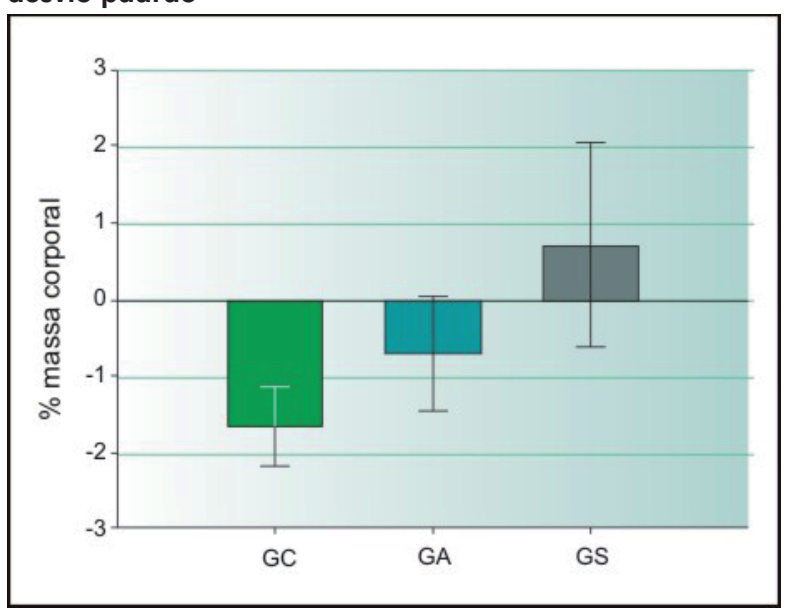

*diferença significativa de GC $(p<0,05)$ 
como da água ${ }^{46}$. No estudo de Grandjean et al. ${ }^{47}$, verificou-se os efeitos na hidratação em homens que foram submetidos a dois diferentes testes de dieta líquida. Em um teste receberam água e em outro não receberam qualquer líquido, não sendo encontradas diferenças na perda de massa corporal entre os grupos. Por outro lado, vários trabalhos expõem que a água pode não ser o melhor repositor de fluidos perdidos durante o exercício. Incluir eletrólitos e carboidratos na reidratação pode melhorar a taxa de absorção intestinal do sódio e da água, assim como repor estoques de glicogênio, aumentando assim o desempenho em exercícios subsequentes ${ }^{34,39,48,49}$. Maughan \& Leiper ${ }^{35}$ afirmam, em seu estudo, que as bebidas esportivas são mais eficazes para a reidratação.

Apesar disso, Noakes ${ }^{23}$ comenta que não há evidências científicas suficientes para recomendação destas bebidas. Na verdade, existe um grande interesse comercial por parte da indústria de bebidas esportivas em lucrar com tais recomendações.

As dúvidas anteriores parecem ser melhor esclarecidas quando a análise do estado de hidratação foi realizada pelo percentual da diferença da massa corporal, na qual o benefício da ingestão da bebida esportiva, no processo de hidratação, fica mais evidente. No atual estudo, o melhor percentual da diferença de perda de massa corporal foi obtido pelo GS, apesar de existir diferença significativa apenas com o GC, e não com GA.

Bergeron et al. ${ }^{\mathbf{2 0}}$, objetivando examinar os efeitos do consumo de bebida esportiva e água na massa corporal de jovens tenistas, também verificaram uma perda significativamente menor na massa corporal quando foi consumida uma bebida esportiva, em uma sessão de treinamento com duração de $120 \mathrm{~min}$. Estes resultados corroboram parcialmente os achados de Ebert et al. ${ }^{50}$, que também encontraram uma variação negativa da massa corporal em jovens ciclistas do sexo masculino, após sessão de treino. Parece que - consumo de água associada a eletrólitos pode, pelo menos, reduzir a quantidade total de líquidos ingeridos que são necessários durante um exercício prolongado ${ }^{51}$.

Os nadadores aracajuanos demonstraram um estado de desidratação quando observados pelo conjunto de marcadores simples de hidratação, ou seja, índice de coloração urinária e percentual da diferença da massa corporal após os treinos. Talvez, a explicação para o fato ocorrido esteja no hábito que os atletas têm de não realizarem uma reposição hídrica nos dias de treinamento. A ingestão de líquidos durante uma sessão de natação é fundamental para manutenção de um adequado estado de hidratação. Porém, apesar do uso da água constituir um bom instrumento, a ingestão de bebida esportiva, parece desempenhar melhor o papel como repositor hídrico. Sugere-se, que os nadadores da cidade de Aracaju ingiram uma quantidade maior de líquidos, antes, durante e após os dias de treinamento, sendo recomendado o uso de bebidas esportivas quando as condições ambientais forem, pelo menos, semeIhantes à deste estudo.

\section{REFERÊNCIAS}

1. Galloway SD. Dehydration, rehydration, and exercise in the heat: rehydration strategies for athletic competition. Can J Appl Physiol. 1999;24(2):188200.

2. Monteiro CR, Guerra I, Barros TL. Hidratação no futebol: uma revisão. Rev Bras Med Esporte. 2003;9(4):238-42.

3. Shirreffs SM. The importance of good hydration for work and exercise performance. Nutr Rev. 2005;63(6):14-21.

4. Aldridge G, Baker JS, Davies B. Effects of hydration status on aerobic performance for a group of male university rugby players. J Exerc Physiol. 2005;8(5):36-42.

5. Cheuvront SN, Haymes EM. Ad libitum fluid intakes and thermoregulatory responses of female distance runners in three environments. J Sports Sci. $2001 ; 19(11): 845-54$.

6. Montain SJ. Hydration recommendations for sport 2008. Curr Sports Med Rep. 2008;7(4):187-92.

7. Sawka MN, Montain SJ. Fluid and electrolyte supplementation for exercise heat stress. Am J Clin Nutr. 2000;72(2 Suppl):S564-72.

8. Maughan RJ. Impact of mild dehydration on wellness and on exercise performance. Eur J Clin Nutr. 2003;57(2 Suppl):S19-23.

9. Maresh CM, Gabaree-Boulant CL, Armstrong LE, Judelson DA, Hoffman JR, Castellani JW, et al. Effect of hydration status on thirst, drinking, and related hormonal responses during low-intensity exercise in the heat. J Appl Physiol. 2004;97(1):39-44.

10. Coyle EF. Cardiovascular drift during prolonged exercise and the effects of dehydration. Int J Sports Med. 1998;19(2 Suppl):S121-4.

11. Murray B. Hydration and physical performance. J Am Coll Nutr. 2007;26(5 Suppl):S542-8.

12. Gonzalez-Alonso J, Crandall CG, Johnson JM. The cardiovascular challenge of exercising in the heat. J Physiol. 2008;586(1):45-53.

13. Montain SJ, Coyle EF. Fluid ingestion during exercise increases skin blood flow independent of blood volume. J Appl Physiol. 1992;73(3):903-10.

14. Gonzalez-Alonso J, Mora-Rodriguez R, Below PR, Coyle EF. Dehydration markedly impairs cardiovascular function in hyperthermic endurance athletes during exercise. J Appl Physiol. 1997;82(4):1229-36.

15. Gonzalez-Alonso J, Calbet JAL, Nielsen B. Muscle blood flow is reduced with dehydration during prolonged exercise in humans. J Physiol. 1998;513(3):895-905

16. Gonzalez-Alonso J, Teller C, Andersen SL, Jensen FB, Hyldig T, Nielsen B. Influence of body temperature on the development of fatigue during prolonged exercise in the heat. J Appl Physiol. 1999;86(3):1032-9.

17. Coyle EF, González-Alonso J. Cardiovascular drift during prolonged exercise: new perspectives. Exerc Sport Sci Rev. $2001 ; 29(2): 88-92$.

18. Convertino VA, Armstrong LE, Coyle EF, Mack GW, Sawka MN, Senay LC $\mathrm{Jr}$, et al. American College of Sports Medicine position stand: exercise and fluid replacement. Med Sci Sports Exerc. 1996;28(1):1-11.

19. Marins JCB, Dantas EHM, Navarro SZ. Diferentes tipos de hidratação durante o exercício prolongado e sua influência sobre o sódio plasmático. Rev Bras Cienc Mov. 2003;11(1):13-22. 
20. Bergeron MF, Waller JL, Marinik EL. Voluntary fluid intake and core temperature responses in adolescent tennis players: sports beverage versus water. Br J Sports Med. 2006;40(5):406-10.

21. Jung AP, Bishop PA, Al-Nawwas A, Dale RB. Influence of hydration and electrolyte supplementation on incidence and time to onset of exerciseassociated muscle cramps. J Athl Train. 2005;40(2):71-5.

22. Aoi W, Naito Y, Yoshikawa T. Exercise and functional foods. Nutr J. 2006;5(15):1-8

23. Noakes TD. Drinking guidelines for exercise: what evidence is there that athletes should drink "as much as tolerable", "to replace the weight lost during exercise" or "ad libitum"?. J Sports Sci. 2007;25(7):781-96.

24. Montain SJ, Coyle EF. Influence of graded dehydration on hyperthermia and cardiovascular drift during exercise. J Appl Physiol. 1992;73(4):1340-50.

25. Oppliger RA, Bartok C. Hydration testing for athletes. Sports Med 2002;32(15):959-71.

26. Brito ISS, Brito CJ, Fabrini SP, Marins JCB. Caracterização das práticas de hidratação em karatecas do estado de Minas Gerais. Fit Perf J. 2006;5(1):23-9.

27. Shirreffs SM. Markers of hydration status. Eur J Clin Nutr. 2003;57(2 Suppl):S6-9.

28. Maughan RJ, Shirreffs SM. Development of individual hydration strategies for athletes. Int J Sport Nutr Exerc Metab. 2008;18(5):457-72.

29. Armstrong LE, Maresh CM, Castellani JW, Bergeron MF, Kenefick RW, LaGasse KE, et al. Urinary indices of hydration status. Int J Sport Nutr. $1994 ; 4(3): 265-79$

30. Armstrong LE, Soto JAH, Hacker FT, Casa DJ, Kavouras SA, Maresh CM. Urinary indices during dehydration, exercise, and rehydration. Int J Sport Nutr. 1998;8(4):345-55.

31. Cheung SS, Mclellan TM. Heat acclimation, aerobic fitness, and hydration effects on tolerance during uncompensable heat stress. J Appl Physiol. 1998;84(5):1731-9.

32. Saat $M$, Sirisinghe RG, Singh R, Tochihara Y. Effects of short-term exercise in the heat on thermoregulation, blood parameters, sweat secretion and sweat composition of tropic-dwelling subjects. J Physiol Anthropol Appl Human Sci. 2005;24(5):541-9.

33. Casa DJ, Armstrong LE, Hillman SK, Montain SJ, Reiff RV, Rich BS, et al. National Athletic Trainer's Association Position Statement: fluid replacement for athletes. J Athl Train. 2000;35(2):212-24.

34. Casa DJ. Exercise in the heat. II. Critical concepts in rehydration, exertional heat ilnesses, and maximizing athletic performance. J Athl Train. $1999 ; 34(3): 253-62$.

35. Maughan RJ, Leiper JB. Fluid replacement requirements in soccer. J Sports Sci. 1994; 12:S29-34

36. Binkley HM, Beckett J, Casa DJ, Kleiner DM, Plummer PE. National Athletic Trainers' Association Position Statement: exertional heat illnesses. J Athl Train. 2002;37(3):329-43.
37. Murray R. Rehydration strategies-balancing substrate, fluid, and electrolyte provision. Int J Sports Med. 1998;19(2 Suppl):S133-5

38. Armstrong LE, Epstein Y, Greenleaf JE, Haymes EM, Hubbard RW, Roberts WO, et al. American College of Sports Medicine position stand: heat and cold illnesses during distance running. Med Sci Sports Exerc. 1996;28(12):39-60.

39. Carvalho T, Rodrigues T, Meyer F, Lancha Jr AH, De Rose EH. Modificações dietéticas, reposição hídrica, suplementos alimentares e drogas: comprovação de ação ergogênica e potenciais riscos para a saúde. Rev Bras Med Esporte. 2003;9(2):43-56.

40. Kutlu M, Guler G. Assessment of hydration status by urinary analysis of elite junior taekwon-do athletes in preparing for competition. J Sports Sci. $2006 ; 24(8): 869-73$

41. Finn JP, Wood RJ. Incidence of pre-game dehydration in athetes competing at an international event in dry tropical conditions. Nutr Diet. $2004 ; 61(4): 221-5$.

42. Yeargin SW, Casa DJ, Armstrong LE, Watson G, Judelson DA, Psathas E, et al. Heat acclimatization and hydration status of American football players during initial summer workouts. J Strength Cond Res. 2006;20(3):463-70.

43. Tan B, Sunarja F. Body mass changes and nutrient intake of optimist class sailors on a race day. J Sports Sci. 2007;25(10):1137-40.

44. Kavouras SA. Assessing hydration status. Curr Opin Clin Nutr Metab Care $2002 ; 5(5): 519-24$.

45. Kovacs EM, Senden JM, Brouns F. Urine color, osmolality and specific electrical conductance are not accurate measures of hydration status during postexercise rehydration. J Sports Med Phys Fitness. 1999;39(1):47-53.

46. Hill RJ, Bluck LJ, Davies PS. The hydration ability of three commercially available sports drinks and water. J Sci Med Sport. 2008;1 1 (2):116-23.

47. Grandjean AC, Reimers KJ, Haven MC, Curtis GL. The effect on hydration of two diets, one with and one without plain water. J American Coll Nutr. $2003 ; 22(2): 165-73$.

48. González-Alonso J, Heaps CL, Coyle EF. Rehydration after exercise with common beverages and water. Int J Sports Med. 1992;13(5):399-406.

49. Stachenfeld NS. Acute effects of sodium ingestion on thirst and cardiovascular function. Curr Sports Med Rep. 2008;7(4 Suppl):S7-13

50. Ebert TR, Martin DT, Stephens B, McDonald W, Withers RT. Fluid and food intake during professional men's and women's road-cycling tours. Int J Sports Phys Perf. 2007;2:58-71.

51. Cuddy JS, Ham JA, Harger SG, Slivka DR, Ruby BC. Effects of an electrolyte additive on hydration and drinking behavior during wilfire suppression. Wilderness Environ Med. 2008;19(3):172-80.

Recebido: 23/10/08 - Aceito: 02/02/09 